\title{
CORRIGENDUM
}

\section{Clinical features and genetic analysis of Korean patients with Loeys-Dietz syndrome}

\author{
Jeong Hoon Yang, Chang-Seok Ki, Hyejin Han, Bong Gun Song, Shin Yi Jang, Tae-Young Chung, Kiick Sung, \\ Heung Jae Lee and Duk-Kyung Kim
}

Journal of Human Genetics (2012) 57, 398; doi:10.1038/jhg.2012.42

Correction to: Journal of Human Genetics (2012) 57, 52-56; doi:10.1038/jhg.2011.130; published online 24 November 2011

Since the publication of the above article, the authors have noticed errors in the 'Results' section (Genetic Analysis) and in descriptions in Figure 1. In the 'Results' section, two of the five novel mutations identified in this study were incorrectly given. Correct sentence should have read as 'Four of the five novel mutations identified in this study were missense mutations in the serine-threonine kinase domain mutations, including p.Asp266Tyr (c.796G $>$ T) and p.Thr375Arg (c.1124C>G) in the TGFBR1, and p.His377Arg (c.1130A>G) and p.Cys514Arg (c.1540 T > C) in the TGFBR2'.

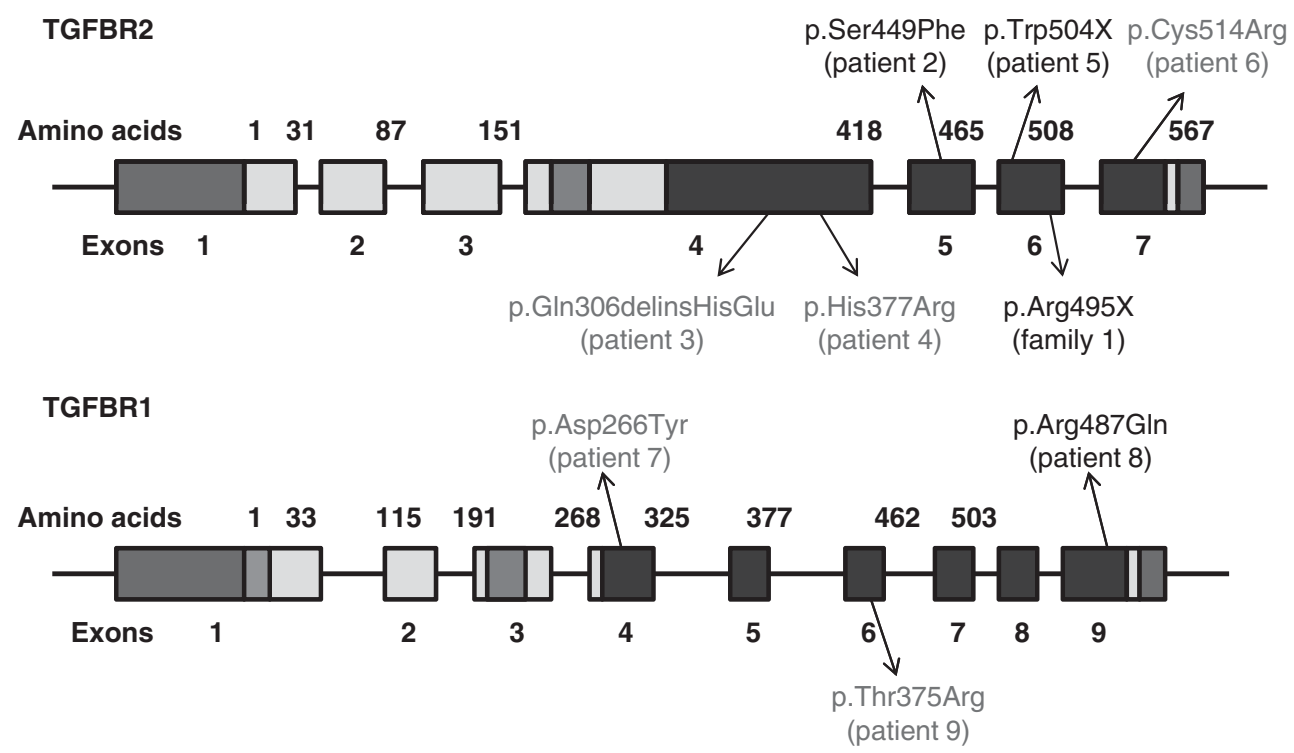

Figure 1 Mutations in the TGFBR2 and the TGFBR1 genes. Novel mutations are indicated in red type. The different exons are numbered and intervening sequences are shown as black lines. The colored boxes represent the exons encoding the extracellular domain of the receptor (yellow), the transmembrane domain (green) and the serine-threonine kinase domain (blue). TGFBR2 mutations were found in seven patients (six probands), and TGFBR1 mutations were found in three patients (three probands). A full color version of this figure is available at the Journal of Human Genetics journal online. 